\title{
Crossover effects in a discrete deposition model with Kardar-Parisi-Zhang scaling
}

\author{
Anna Chame and F. D. A. Aarão Reis \\ Instituto de Física, Universidade Federal Fluminense, \\ Avenida Litorânea s/n, 24210-340 Niterói RJ, Brazil
}

(November 23, 2018)

\begin{abstract}
We simulated a growth model in $1+1$ dimensions in which particles are aggregated according to the rules of ballistic deposition with probability $p$ or according to the rules of random deposition with surface relaxation (Family model) with probability $1-p$. For any $p>0$, this system is in the KardarParisi-Zhang $(K P Z)$ universality class, but it presents a slow crossover from the Edwards-Wilkinson class $(E W)$ for small $p$. From the scaling of the growth velocity, the parameter $p$ is connected to the coefficient of the nonlinear term of the $K P Z$ equation, $\lambda$, giving $\lambda \sim p^{\gamma}$, with $\gamma=2.1 \pm 0.2$. Our numerical results confirm the interface width scaling in the growth regime as $W \sim \lambda^{\beta} t^{\beta}$ and the scaling of the saturation time as $\tau \sim \lambda^{-1} L^{z}$, with the expected exponents $\beta=1 / 3$ and $z=3 / 2$ and strong corrections to scaling for small $\lambda$. This picture is consistent with a crossover time from $E W$ to $K P Z$ growth in the form $t_{c} \sim \lambda^{-4} \sim p^{-8}$, in agreement with scaling theories and renormalization group analysis. Some consequences of the slow crossover in this problem are discussed and may help investigations of more complex models.
\end{abstract}

PACS numbers: 05.40.-a, 05.50.+q 


\section{INTRODUCTION}

Surface growth processes and deposition of thin films are of great interest due to potential technological applications (such as production of nanostructures for microelectronic devices, the possibility of growth of designed quantum objects, magnetic storage devices, among others) and due to the fundamental role these systems play in non-equilibrium statistical physics [1,2]. Several models have been investigated in the last decade, most of them involving one kind of particle and a simple microscopic aggregation rule. The competition between different growth mechanisms have received less attention, but is essential to describe some practical situations, such as growth of materials designed to have specific electronic, mechanical or magnetic properties, which involves deposition of two or more chemical species. In this framework, some authors considered growth models with two kinds of particles and different aggregation rules [3, [4,5]. Other situations involving competition between two growth mechanisms have also been considered [6,7, 8].

These models usually show crossover effects from one dynamics at small times $t$ or short length scales $L$ to another dynamics at long $t$ and large $L$. One typical example is KardarParisi-Zhang (KPZ) growth at small nonlinearities [9]. The Langevin-type equation

$$
\frac{\partial h}{\partial t}=\nu \nabla^{2} h+\frac{\lambda}{2}(\nabla h)^{2}+\eta(\vec{x}, t)
$$

known as $K P Z$ equation, was proposed as a hydrodynamic description of kinetic surface roughening. Here $h$ is the height at the position $\vec{x}$ in a $d$-dimensional substrate at time $t, \nu$ represents a surface tension, $\lambda$ represents the excess velocity and $\eta$ is a Gaussian noise [1,9]

with zero mean and variance $\left\langle\eta(\vec{x}, t) \eta\left(\overrightarrow{x^{\prime}}, t^{\prime}\right)\right\rangle=D \delta^{d}\left(\vec{x}-\overrightarrow{x^{\prime}}\right) \delta\left(t-t^{\prime}\right)$. When the coefficient $\lambda$ of the nonlinear term is small, a crossover is observed from linear growth $(\lambda=0$, known as Edwards-Wilkinson theory - EW) [10] to $K P Z$ behavior.

In discrete models, the interface width, which characterizes the roughness of the interface, is defined as

$$
W(L, t)=\left[\left\langle\frac{1}{L^{d}} \sum_{i}\left(h_{i}-\bar{h}\right)^{2}\right\rangle\right]^{1 / 2}
$$

for deposition in a $d$-dimensional substrate of length $L\left(h_{i}\right.$ is the height of column $i$ at time $t$, the bar in $\bar{h}$ denotes a spatial average and the angular brackets denote a configurational average). For short times it scales as $W \sim t^{\beta}$ and for long times, in the steady state regime, it saturates at $W_{\text {sat }} \sim L^{\alpha}$. The dynamical exponent $z=\alpha / \beta$ characterizes the crossover from the growth regime to the steady state regime. For systems belonging to the $E W$ universality class, we have $\alpha_{0}=1 / 2, \beta_{0}=1 / 4$ and $z_{0}=2$ in $d=1$ (in this paper, the subscript 0 will refer to exponents of the $E W$ theory). For systems in the $K P Z$ class, in $d=1$, we have $\alpha=1 / 2, \beta=1 / 3, z=3 / 2$ [1, 9,10$]$.

Considering the crossover from $E W$ to $K P Z$ scaling in $d=1$, Grossmmann, Guo and Grant $(G G G)$ [11] and Nattermann and Tang $(N T)$ [12] (see also the review by Forrest and Toral [13]) proposed multiscaling relations that are equivalent to

$$
W(L, t)=L^{\alpha} f\left(\frac{t}{t_{c}}, \frac{L}{\xi_{c}}\right),
$$


in which $\xi_{c} \sim t_{c}^{1 / z_{0}}$. GGG also proposed that the characteristic time of crossover from $E W$ to $K P Z$ dynamics was

$$
t_{c} \sim \lambda^{-\phi},
$$

with $\phi>0$, since the $E W-K P Z$ crossover disappears for $\lambda=0$. Through scaling arguments, those authors obtained $\phi=z_{0} /\left(\alpha_{0}+z_{0}-2\right)$, which gives $\phi=4$ in $d=1$. This was confirmed through one-loop renormalization group calculations by $N T$. The scaling analysis of the $K P Z$ equation by Amar and Family $(A F)$ [14] and the assumption of Family-Vicsek scaling [15] were used to show that, in the nonlinear and saturation regimes,

$$
W(L, t) \sim L^{1 / 2} g\left(|\lambda| \frac{t}{L^{3 / 2}}\right),
$$

in which $g$ is a scaling function and the dependence of $W$ on the parameters $\nu$ and $D$ of Eq. (II) was omitted. A generalized scaling relation equivalent to Eq. (3), which is a more general result than Eq. (5), was also obtained by Derrida and Mallick in the context of the connection to the one-dimensional asymmetric exclusion model [16]. Amar and Family [14] have shown that the scaling form (5) also predicts a crossover exponent $\phi=4$.

On the other hand, all previous numerical results suggested $\phi \approx 3$; for instance, $G G G$ obtained this value using data collapse methods [11]. Thus, it would be desirable to confirm numerically the scaling properties predicted for a $K P Z$ system in order to solve this controversy.

The purpose of this work is to study a competitive growth process with $E W$ to $K P Z$ crossover, involving ballistic deposition $(B D)$ [1, 17] and random deposition with surface relaxation (Family model) [18 in $d=1$. In this model, incident particles aggregate to the deposit according to the rules of $B D$ with probability $p$ and according to the rules of the Family model with probability $1-p$. It is known that the Family model is in the $E W$ universality class, while $B D$ is in the $K P Z$ class. This competitive model was introduced by Pellegrini and Jullien [19], whose main interest was the connection to the roughening transition present in higher dimensions. Although it is expected that this model is in the $K P Z$ class for any $p>0$, the crossover in $d=1$ was not studied in detail in their original work and, for $p \lesssim 0.3$, effective exponents very near the $E W$ values were obtained [19].

Here we will simulate that model in order to analyze the interface width scaling in the nonlinear regime, the crossover to the saturation regime and to connect the parameter $p$ and the coefficient $\lambda$ of the $K P Z$ equation in the corresponding continuum limit. The amplitudes of typical saturation times and of interface width scaling in the growth regime are consistent with multiscaling concepts [11,12,14 and refine previous numerical estimates for related systems. The crossover exponent $\phi=4$ follows directly from our numerical results and, together with the observed relation $\lambda \sim p^{2.1}$, indicate that the crossover at small $p$ is very slow. The analysis of this apparently simple problem shows that, in order to obtain reliable asymptotic exponents governing various quantities, it is essential to account for corrections to the leading terms in the scaling relations. Thus, this work may also be relevant to the analysis of other systems with slow crossover to $K P Z$ scaling, whose interest increased after the recent debate on the problem of Fisher waves and their discrete realizations in $d=1$ dimensions [20,21,22,23]. For that reason, the crossover effects identified in our simulations' data will be discussed in detail. 
It is also relevant to point out that a related competitive model was recently studied in $d=1$ and $d=2$ [24], showing evidence of the asymptotic $K P Z$ behavior. However, that work did not study the relation between the parameters of the discrete and the continuous $(K P Z)$ model nor the scaling amplitudes that will be considered here.

The rest of this work is organized as follows. In Sec. II, we will define precisely the discrete model and connect it to the $K P Z$ equation using the scaling properties of the growth velocity. In Secs. III and IV we will present results for the interface width scaling in the discrete model at the nonlinear growth regime and at the steady state regime, respectively. In Sec. V we summarize our results and present our conclusions.

\section{THE DISCRETE MODEL AND ITS CONNECTION TO THE $K P Z$ THEORY}

We considered a model in which particles are aggregated following the rules of $B D$ with probability $p$ or the rules of random deposition with surface relaxation (Family model) with probability $1-p$. In $B D$ (Fig. 1a), the incident particle follows a straight trajectory perpendicular to the surface and sticks upon first contact with a nearest neighbor occupied site. It leads to the formation of a porous deposit. In the Family model (Fig. 1b), the particle falls towards the surface along the incident column and sticks at the top of that column if its height is lower than or equal to the heights of the neighboring columns. Otherwise, the particle diffuses to the neighboring column which has the lowest height and, if two or more neighbors have the same height, it chooses one of them randomly.

For $p=0$, we have the Family model, which is in the $E W$ universality class. For any $p \neq 0$, in $d=1$, we expect the $B D$ process to change the universality class to $K P Z$ in the continuum limit (see the analysis in Ref. [25] for a related model). Then the coefficient $\lambda$ of the nonlinear term vanishes with $p$ in the form

$$
\lambda \sim p^{\gamma}
$$

with $\gamma>0$ (to be estimated below). For small $p$ and sufficiently large $L$, the interface width $W(L, t, p)$ must scale analogously to the weak coupling regime of the KPZ theory [13, 19, in which three regimes were identified: a linear $(E W)$ growth regime at early times $\left(t \ll t_{c}\right)$, a nonlinear $(K P Z)$ growth regime for $t_{c} \ll t \ll \tau$ and the saturation regime for $t \gg \tau$, as illustrated in Fig. 2 ( $\tau$ is the characteristic time for the interface width saturation).

In order to calculate the exponent $\gamma$, we considered the scaling of the interface growth velocity. The difference between the growth velocity in an infinitely large substrate, $v_{\infty}$, and the velocity in the steady state of a finite lattice (thick films), $v(L)$, scales as [26,27]

$$
\Delta v(L) \equiv v_{\infty}-v(L) \sim \lambda L^{-\alpha_{\|}}
$$

with $\alpha_{\|}=1$ in $d=1$ [26]. Defining

$$
b_{v}(L) \equiv \Delta v(L) \times L,
$$

we expect that, as $L \rightarrow \infty$,

$$
b_{v}(L) \rightarrow B_{v}=B \lambda,
$$


where $B$ is a constant.

In the discrete model, $\lambda$ varies with $p$, consequently $b_{v}$ is a function of $L$ and $p$ which has a finite limiting value $B_{v}(p)$ as $L \rightarrow \infty$. For very large $L$, Eqs. (6) and (9) shows that $B_{v}$ scales with $p$ with exponent $\gamma$.

Simulations of the model were performed in lattice of lengths from $L=16$ to $L=4096$ until the saturation regime, and in lattices with $L=2^{16}=65536$ during the growth regime (linear and nonlinear), for several values of the probability $p$ between $p=0.15$ and $p=0.5$. The results presented in this paper are averages typically over $10^{5}$ realizations for the smallest lattices $(L \leq 256), 10^{4}$ realizations for $256 \leq L \leq 4096$ and $10^{2}$ realizations for $L=65536$. The growth velocities were calculated from numerical derivatives of the average heights of the deposits, with accuracies from 5 to 6 decimal places, in lattices of lengths $L \leq 128$ ( $L \leq 512$ for $p=0.15$ ). We considered the data for $L=65536$ as representative of an infinite lattice in the growth regime (some simulations in $L=131072$ supported this assumption), and also obtained $v_{\infty}$ with high accuracy. These data provided estimates of $b_{v}(p, L)$ with accuracy from $0.5 \%$ to $5 \%$. For larger lengths, poorer results were obtained due to the much smaller number of realizations.

In Fig. 3 we show $b_{v}(p, L)$ versus $1 / L$ for the three smallest values of $p$ considered in this work. The variable $1 / L$ in the abscissa was the best choice to represent finite-size corrections in $b_{v}$ as $L \rightarrow \infty$, and is related to higher order terms $\left(1 / L^{2}\right)$ in Eq. (đ). Such scaling corrections have been previously observed in the analysis of small $L$ data for $B D$ and for the restricted solid-on-solid model by Krug and Meakin [26]. The corrections were considered in the extrapolation of the data in Fig. 3, which provided estimates of $B_{v}(p)$ for several values of $p$ (intercepts with the vertical axis in Fig. 3).

Crossover effects may be crucial in the extrapolation procedure discussed above, and may severely affect the estimates of $B_{v}$ for small $p$. For $0.25 \leq p \leq 0.5$, four values of $b_{v}$ $(16 \leq L \leq 128)$ were well fitted by straight lines in the $b_{v}(p, L) \times 1 / L$ plots (these results were not shown in Fig. 3, except for $p=0.25)$. For $p=0.2$, the data for $32 \leq L \leq 256$ confirm the presence of the $1 / L$ correction and was also used to estimate $B_{v}$ (the estimate for $L=16$ deviates from this trend). On the other hand, for $p=0.15$, the result for $L=256$ showed a crossover in $b_{v}$, which suggested calculations for $L=512$. Fig. 3 shows that $b_{v}(0.15, L)$ slowly increases for $16 \leq L \leq 128$, but decreases for $128 \leq L \leq 512$. Consequently, the extrapolation considered only the three last points (see Fig. 3) and gave $B_{v} \approx 0.057$. However, if the extrapolation to $L \rightarrow \infty$ was performed only with results for $L \leq 128$, then a $7 \%$ larger value of $B_{v}$ would be obtained. Smaller values of $p$ were not studied here because such crossover would appear for much larger $L$ and, consequently, the extrapolations based on small systems' data would provide unreliable estimates of $b_{v}(p, \infty)$.

In Fig. 4 we show $\ln \left[B_{v}(p)\right]$ versus $\ln p$ using the extrapolated values of $B_{v}$, as discussed above. The linear fit in Fig. 4 gives $B_{v} \sim p^{2.1}$. Considering the error bars in $B_{v}$, we obtain an exponent $\gamma=2.1 \pm 0.2$ (Eq. 6).

The large value of the exponent $\gamma$ explains the crossover effect discussed above. Since $\lambda$ decreases rapidly with $p$, the coefficient of the leading term in $\Delta v$ (Eq. (7) is small compared to higher order corrections $\left(1 / L^{2}, 1 / L^{3}\right.$ etc) for small $p$. Thus, very large values of $L$ are needed to provide reliable extrapolations with a single correction term, which prevented us to use values of $p<0.15$ in our study. 


\section{INTERFACE WIDTH AT THE NONLINEAR GROWTH REGIME}

In the nonlinear growth regime $\left(t_{c} \ll t \ll \lambda^{-1} L^{z}\right)$ for sufficiently large substrates in $d=1$, the interface width does not depend on $L$ (weak finite-size effects). Then the scaling function of Eq. (5) behaves as

$$
g(x) \approx C x^{\beta}, \beta=1 / 3,
$$

with constant $C$, so that $W$ does not depend on $L$, except for vanishing corrections to scaling. Consequently, the $\lambda$-dependent scaling of $W$ in this regime is

$$
W \approx C \lambda^{\beta} t^{\beta} .
$$

In this section, we will verify this $\lambda$-dependence through a careful analysis of simulations' data of our discrete model.

However, first we will show that Eq. (11) gives $\phi=4$ (Eq. (1) in a simple way, as follows. The crossover $E W-K P Z$ (at $t \sim t_{c}$ ) occurs when the scaling relation (11) matches the $E W$ scaling

$$
W(t, L) \approx C^{\prime} t^{\beta_{0}},
$$

with $C^{\prime}$ constant. Thus

$$
t_{c}^{\beta_{0}} \sim \lambda^{\beta} t_{c}{ }^{\beta},
$$

then we obtain $\phi=\frac{\beta}{\left(\beta-\beta_{0}\right)}=\frac{z_{0}}{\left(z_{0}-z\right)}=4$.

We conclude that the numerical test of Eq. (11), in particular of the dependence on the parameter $\lambda$, may be used to test the proposal $\phi=4$. The first step is to extract the amplitude of $t^{\beta}$ scaling in Eq. (11), which motivates the definition of the amplitude $a(p, t)$ as

$$
a(p, t) \equiv W(L \rightarrow \infty, t) / t^{1 / 3} .
$$

In Fig. 5a we show $a(p, t)$ versus $1 / t^{1 / 3}$ for several values of $p$, using the data for $L=$ $2^{16}=65536$. Different variables in the form $1 / t^{x}(x>0)$ were tested in the abscissa, but the variable $1 / t^{1 / 3}$ of Fig. 5a provided the best linear fits for most values of $p$. The fact that in Fig. 5a $a(p, t)$ is still decreasing for large $t$ indicates the presence of a constant (independent of $t$ ) correction to the leading behavior in Eq. (11). It proves again the relevance of accounting for scaling corrections in this problem, although we are not able to justify these corrections on theoretical grounds.

As $t \rightarrow \infty, a(p, t)$ converges to a finite limiting value

$$
A(p) \equiv a(p, \infty) .
$$

$A(p)$ is the complete amplitude of $t^{\beta}$ scaling of the interface width in the nonlinear growth regime (Eq. 11). Our estimates of $A(p)$ were obtained from linear extrapolations of $a(p, t) \times$ $1 / t^{1 / 3}$ plots to $t \rightarrow \infty$ (intercepts with the vertical axis in Fig. 5a).

From Eq. (11), it is expected that the amplitude $A(p)$ scales as $\lambda^{\beta}$. From the connection relation $($ 可), it is expected that 


$$
A(p) \sim p^{\delta}
$$

with

$$
\delta=\gamma \beta
$$

Then, the test of Eq. (11) reduces to the test of Eq. (17) for the amplitude exponent $\delta$.

In order to calculate the exponent $\delta$ in the relation (16), our first step was to plot $\log [A(p)]$ versus $\log p$, but we noticed that it showed decreasing slopes as $p$ decreased. We analyzed the evolution of the slopes of $\log [A(p)] \times \log p$ plots by calculating the following effective exponents for consecutive values $p=p^{\prime}$ and $p=p^{\prime \prime}$ :

$$
\delta_{p}=\frac{\ln \left[a\left(p^{\prime}, \infty\right) / a\left(p^{\prime \prime}, \infty\right)\right]}{\ln \left(p^{\prime} / p^{\prime \prime}\right)}, p=\sqrt{p^{\prime} p^{\prime \prime}}
$$

so that, as $p \rightarrow 0(\lambda \rightarrow 0)$, we expect that $\delta_{p} \rightarrow \delta$.

In Fig. $5 \mathrm{~b}$ we show $\delta_{p}$ versus $p^{2}$, which gives a reasonable linear fit and indicates that $\delta=0.7 \pm 0.2$. Again the variable $p^{2}$ in the abscissa is the one that provides the best linear fit of the central estimates of $\delta_{p}$, chosen among other variables in the form $p^{y}(y>0)$. In Fig. 5b, the effective exponents systematically decrease as $p$ decreases, which reflects our previous observation of decreasing slopes in $\log [A(p)] \times \log p$ plots.

Our estimates $\delta=0.7 \pm 0.2$ and $\gamma=2.1 \pm 0.2$ (Sec. III) are consistent with relation (17) with $\beta=1 / 3$. Even considering that the error bars are large, it is relevant to notice that the central estimates confirm that relation exactly, which gives additional support to our analysis.

\section{INTERFACE WIDTH NEAR AND AT THE STEADY STATE REGIME}

Our numerical results in the steady state regime provide additional support for the scaling picture proposed for the problem.

From Eq. (5), we expect that the crossover from the nonlinear to the steady state regime takes place at a characteristic time $\tau$ that scales as

$$
\tau \sim \lambda^{-1} L^{z}
$$

with $z=3 / 2$ in $d=1$. In this section, our main purpose is to test the $\lambda$-dependence of this characteristic time in our discrete model.

The saturation time $\tau$ is usually estimated using some arbitrary recipe. Here, instead of estimating the saturation time $\tau$ (which may be defined from the time dependence of the interface width as it converges to the saturation value), we calculated a characteristic time $\tau_{0}$ which is proportional to $\tau$, according to a recently proposed method [28]. That method provided accurate estimates of dynamic exponents for several growth models in $d=1$ and $d=2$, including the Family and the $B D$ models.

First, the saturation width $W_{s}$ is estimated, for fixed $p$ and $L$. Then we define $\tau_{0}$ through

$$
W\left(\tau_{0}\right)=k W_{s},
$$


with fixed $k(k \lesssim 1)[28]$. Using the Family-Vicsek relation $W(L, t)=L^{\alpha} f\left(t L^{-z}\right)$ and considering that $W_{s} \sim L^{\alpha}$, we conclude that $\tau_{0} \sim L^{z}$, i.e., $\tau_{0}$ is proportional to the saturation time $\tau$. For the particular case of a $K P Z$ system, Eq. (5) gives

$$
\tau_{0} \sim \lambda^{-1} L^{3 / 2}
$$

Extending the procedure of previous work [28], we considered $k=1-1 / e=0.6321 \ldots$ in Eq. (20) to estimate $\tau_{0}$. This value of $k$ gave $\tau_{0} \approx \tau$ for $B D$, where $\tau$ was estimated from the decay of $W_{s}-W$ [28]. In the present model, for fixed $p$, we calculated the ratios $\tau_{0} / L^{3 / 2}$ for several lengths $L$ and obtained the asymptotic amplitude

$$
D(p)=\frac{\tau_{0}}{L^{3 / 2}}, \quad L \rightarrow \infty .
$$

The extrapolation procedure follows the same lines of the calculation of $A(p)$ from $a(p, t)$ in Sec. III]. However, only results for $p \geq 0.2$ could be obtained using data for lattice sizes $L \leq 4096$, since the saturation for smaller values of $p$ is typically of $E W$ type $\left(\tau \sim L^{2}\right)$ in this range of $L$.

From Eqs. (22) and (21), we expect that $D(p) \sim \lambda^{-1}$. Consequently, it must scale with $p$ as

$$
D(p) \sim p^{-\gamma} .
$$

In Fig. 6 we show $\ln D(p)$ versus $\ln p$, with a linear fit that gives $D(p) \sim p^{-2.1}$. This result is consistent with the independent estimate of $\gamma$ from Eq. (6) (Sec. III).

We also analyzed the scaling of the saturation width $W_{s}$. For lattice sizes $L \leq 1024$, we obtained $W_{s} \sim L^{\alpha}$ with $\alpha=1 / 2$ and weak corrections to scaling. Using the data for $L=1024$, we defined

$$
\Delta W_{s} \equiv W_{s}(p)-W_{s}(0)
$$

as the difference between the saturation width for a given probability $p$ and the saturation width for the Family model $(p=0)$.

In Fig. 7 we show $\ln \Delta W_{s}$ versus $\ln p$. The linear fit suggests $\Delta W_{s} \sim p^{3 / 2}$, thus we obtain the complete form for the saturation width as

$$
W_{s} \approx\left(C_{1}+C_{2} p^{3 / 2}\right) L^{\alpha}
$$

with $\alpha=1 / 2, C_{1}$ and $C_{2}$ constants. The amplitude of $W_{s}$ scaling is $\left(\frac{D}{24 \nu}\right)^{1 / 2}$ [27], i.e., the heights' fluctuations depend only on the parameters $\nu$ and $D$ of the $K P Z$ equation (1), but not on the nonlinearity parameter $\lambda$. Thus we conclude that the dependence on $p$ in Eq. (25) is related to the dependence on $p$ of the surface tension parameter $\nu$ : when $p$ decreases, the amplitude in Eq. (25) decreases, then the parameter $\nu$ increases. Indeed, this term is physically expected to increase in the crossover from $B D($ low $\nu)$ to the Family model (high $\nu)$. 


\section{SUMMARY AND CONCLUSIONS}

We studied a competitive growth model in $1+1$ dimensions involving two dynamics: ballistic deposition with probability $p$ and random deposition with surface relaxation (Family model) with probability $1-p$. This model is a discrete realization of the continuum $K P Z$ equation with an adjustable nonlinear coupling $\lambda$ related to $p$. At the critical probability $p_{c}=0$, the process belongs to the $E W$ universality class, while any finite value of $p$ drives the system to $K P Z$ class.

We established the connection between the parameters $p$ and $\lambda$ as $\lambda \sim p^{2.1}$ and showed that $W \sim p^{0.7} t^{1 / 3}$ in the growth regime. This indicates that the discrete model presents a very slow crossover from $E W$ to $K P Z$ scaling at small values of $p$, since the crossover time is $t_{c} \sim \lambda^{-4} \sim p^{-8.4}$. This slow crossover explains the discrepancies in the effective exponents $\beta$ measured in that regime in previous works [19].

We also obtained the saturation time $\tau \sim p^{-2.1} L^{3 / 2}$. The condition $\tau \gg t_{c}$ is necessary to observe the crossover to $K P Z$ scaling, while the opposite condition leads to $E W$ saturation without an intermediate $K P Z$ growth of the interface width. A critical system size $\xi_{c}$ separates systems which present $E W$ or $K P Z$ saturation, and $\xi_{c}$ can be estimated from the condition $\tau \sim t_{c}$, which gives $\xi_{c} \sim \lambda^{-2} \sim p^{-4.2}$. This large exponent proves that simulations in very large system sizes are necessary in order to observe all features of $K P Z$ scaling for small $p$.

Our results are consistent with the scaling theories for the weak coupling regime of the $K P Z$ equation proposed by several authors and refine previous numerical analysis. Then we expect that the methods presented here may be helpful to analyze other growth models with slow crossovers to $K P Z$ scaling, in which scaling theories cannot be easily developed. 


\section{REFERENCES}

[1] A.L. Barabási and H.E. Stanley, Fractal concepts in surface growth (Cambridge University Press, Cambribge, England, 1995).

[2] A. Pimpinelli and J. Villain, Physics of Crystal Growth (Cambridge University Press, 1998).

[3] W. Wang and H. A. Cerdeira, Phys.Rev. E 473357 (1993); H. F. El-Nashar and H. A. Cerdeira, Phys. Rev. E 616149 (2000).

[4] F.D.A. Aarão Reis, Phys. Rev. E 66, 027101 (2002).

[5] M. Kotrla, J. Krug and P. Smilauer, Phys. Rev. B 62, 2889 (2000).

[6] C.M. Horowitz and E. Albano, J. Phys. A: Math. Gen. 34357 (2001).

[7] C. M. Horowitz, R.A. Monetti, E.V. Albano, Phys. Rev. E 6366132 (2001).

[8] T. J. da Silva and J. G. Moreira, Phys. Rev. E 63, 041601 (2001).

[9] M. Kardar, G. Parisi and Y.-C. Zhang, Phys. Rev. Lett. 56889 (1986).

[10] S.F. Edwards and D.R. Wilkinson, Proc. R. Soc. London 38117 (1982).

[11] B. Grossmann, H. Guo, and M. Grant, Phys. Rev. A 431727 (1991).

[12] T. Nattermann and L.-H. Tang, Phys. Rev. A 457156 (1992).

[13] B.M. Forrest and R. Toral, J. Stat. Physics, 70, 703 (1993).

[14] J.G. Amar and F. Family, Phys. Rev. A 45 R3373 (1992).

[15] F. Family and T. Vicsek, J. Phys. A 18 L75 (1985).

[16] B. Derrida and K. Mallick, J. Phys. A: Math. Gen. 30, 1031 (1997).

[17] M. J. Vold, J. Coll. Sci. 14168 (1959); J. Phys. Chem. 631608 (1959).

[18] F. Family, J. Phys. A 19 L441 (1986).

[19] Y. P. Pellegrini and R. Jullien, Phys. Rev. Lett. 641745 (1990); Y. P. Pellegrini and R. Jullien, Phys. Rev. A 43920 (1991).

[20] J. Riordan, C. R. Doering and D. ben-Avraham, Phys. Rev. Lett 75565 (1995).

[21] G. Tripathy, A. Rocco, J. Casademunt and W. van Saarloos, Phys. Rev. Lett. 865215 (2001).

[22] R. A. Blythe and M. R. Evans, Phys. Rev. E 64, 051101 (2001).

[23] E. Moro, Phys. Rev. Lett. 87, 238303 (2001).

[24] T. J. da Silva and J. G. Moreira, cond-mat/0207614 (2002).

[25] H. Yan, D. Kessler and L.M. Sander, Phys. Rev. Lett. 64926 (1990).

[26] J. Krug and P. Meakin, J. Phys. A: Math. Gen. 23 L987 (1990).

[27] J. Krug, P. Meakin, and T. Halpin-Healy, Phys. Rev. A 45638 (1992).

[28] F. D. A. Aarão Reis, to appear in Physica A (2002). 


\section{FIGURES}

FIG. 1. (a) The aggregation rules of ballistic deposition, in which the sticking position of each incident particle is marked with a cross. (b) The aggregation rules of the Family model, in which the relaxation of incident particles to their sticking positions is indicated by arrows. The incident particle at the right has equal probabilities to stick at any one of the neighboring columns.

FIG. 2. For small values of $p$ and sufficiently large $L$, the interface width $W(t, L, p)$ presents three regimes: a linear $(E W)$ growth regime at early times $\left(t \ll t_{c}\right)$, a nonlinear $(K P Z)$ growth regime for $t_{c} \ll t \ll \tau$ and the saturation regime for $t \gg \tau$.

FIG. 3. $b_{v}(p, L) \equiv \Delta v \times L$ versus $1 / L$ for $p=0.25$ (squares), $p=0.2$ (triangles) and $p=0.15$ (crosses). Solid lines are least squares fits of the data for larger $L$.

FIG. 4. Log-log plot of $B_{v}(p)$ as a function of $p$. The linear fit gives an exponent $\gamma=2.1 \pm 0.2$.

FIG. 5. (a) Interface width amplitude in the nonlinear regime $a(p, t) \equiv W(L \rightarrow \infty, t) / t^{1 / 3}$ as a function of $1 / t^{1 / 3}$ for $p=0.4, p=0.3, p=0.25, p=0.2$ and $p=0.15$ (from top to bottom); (b) Effective exponent $\delta_{p}$ versus $p^{2}$, with a linear fit that gives the exponent $\delta \approx 0.7$ as $p \rightarrow 0$.

FIG. 6. Log-log plot of the amplitude $D(p)\left(\tau_{0} / L^{3 / 2}\right.$ as $\left.L \rightarrow \infty\right)$ as a function of $p$. The linear fit gives $D(p) \sim p^{-\omega}$ with $\omega \approx 2.1$.

FIG. 7. $\log -\log$ plot of $\Delta W_{s} \equiv W_{s}(p)-W_{s}(0)$ as a function of $p$, using data for $L=1024$. The linear fit suggests $\Delta W_{s} \sim p^{3 / 2}$. 


$$
\begin{aligned}
& \text { 모 } \\
& \square \square \square \\
& \text { 媨 } \\
& \text { 窝 } \\
& \text { (a) } \\
& \text { (b) }
\end{aligned}
$$




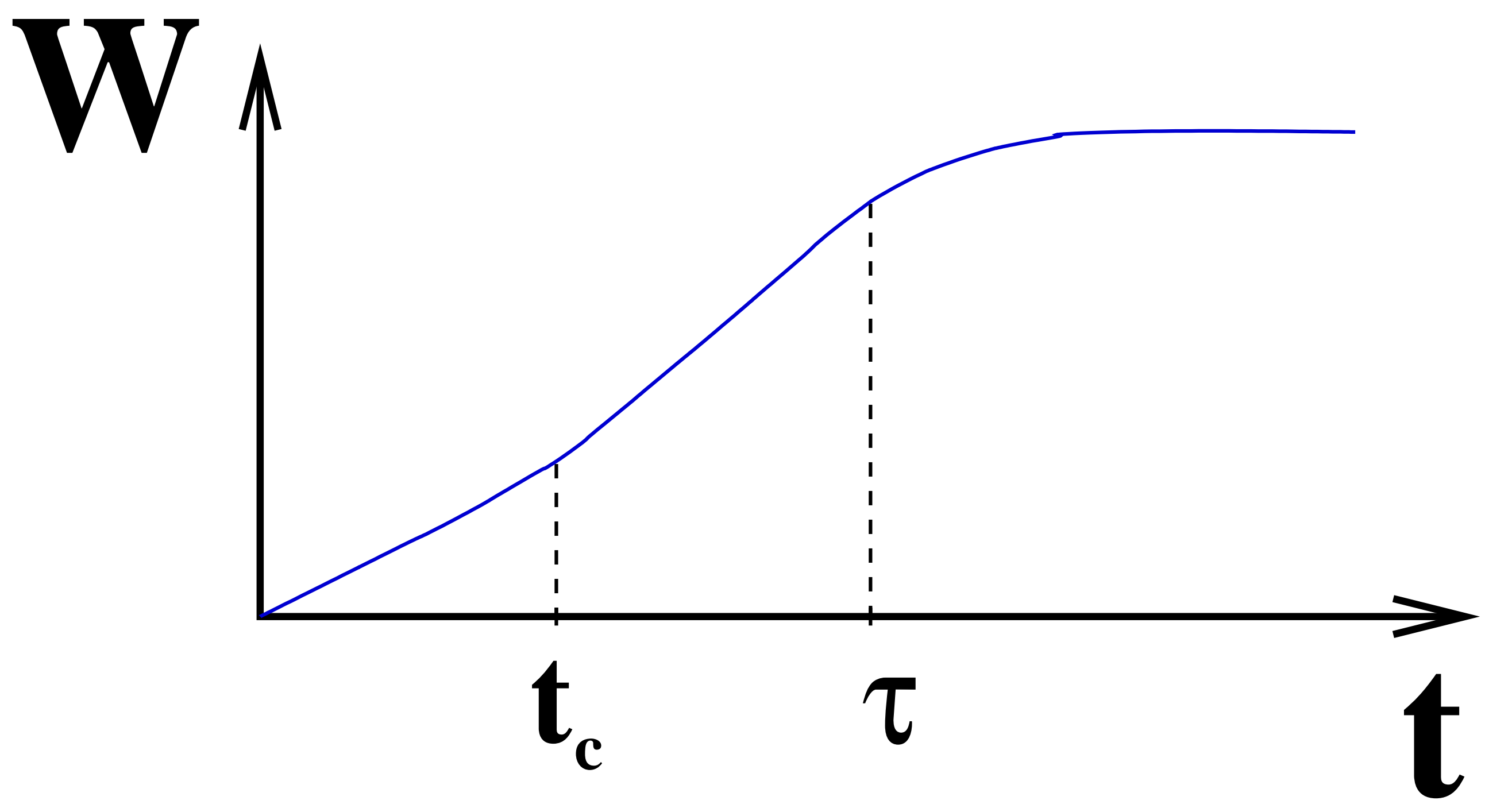




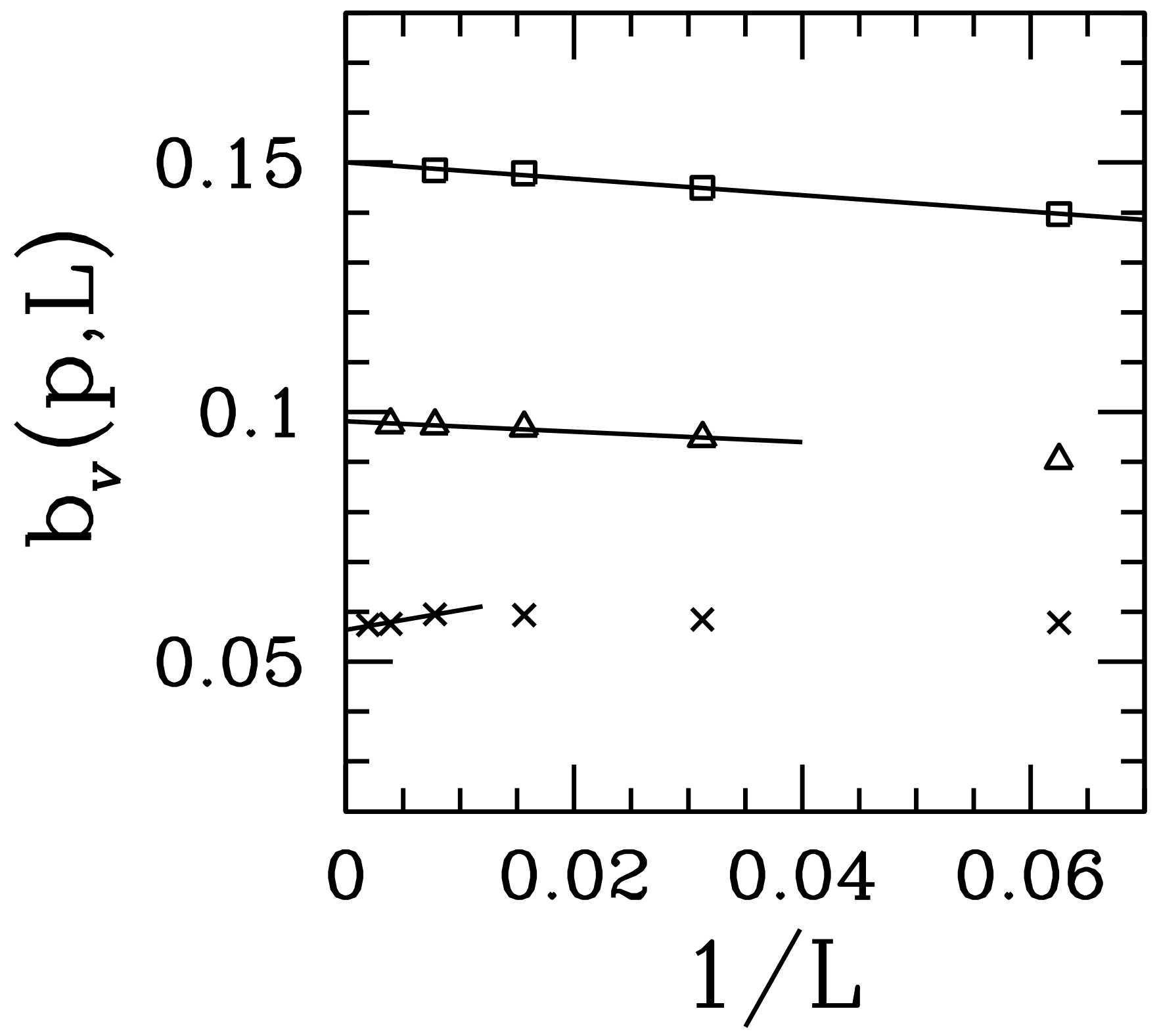




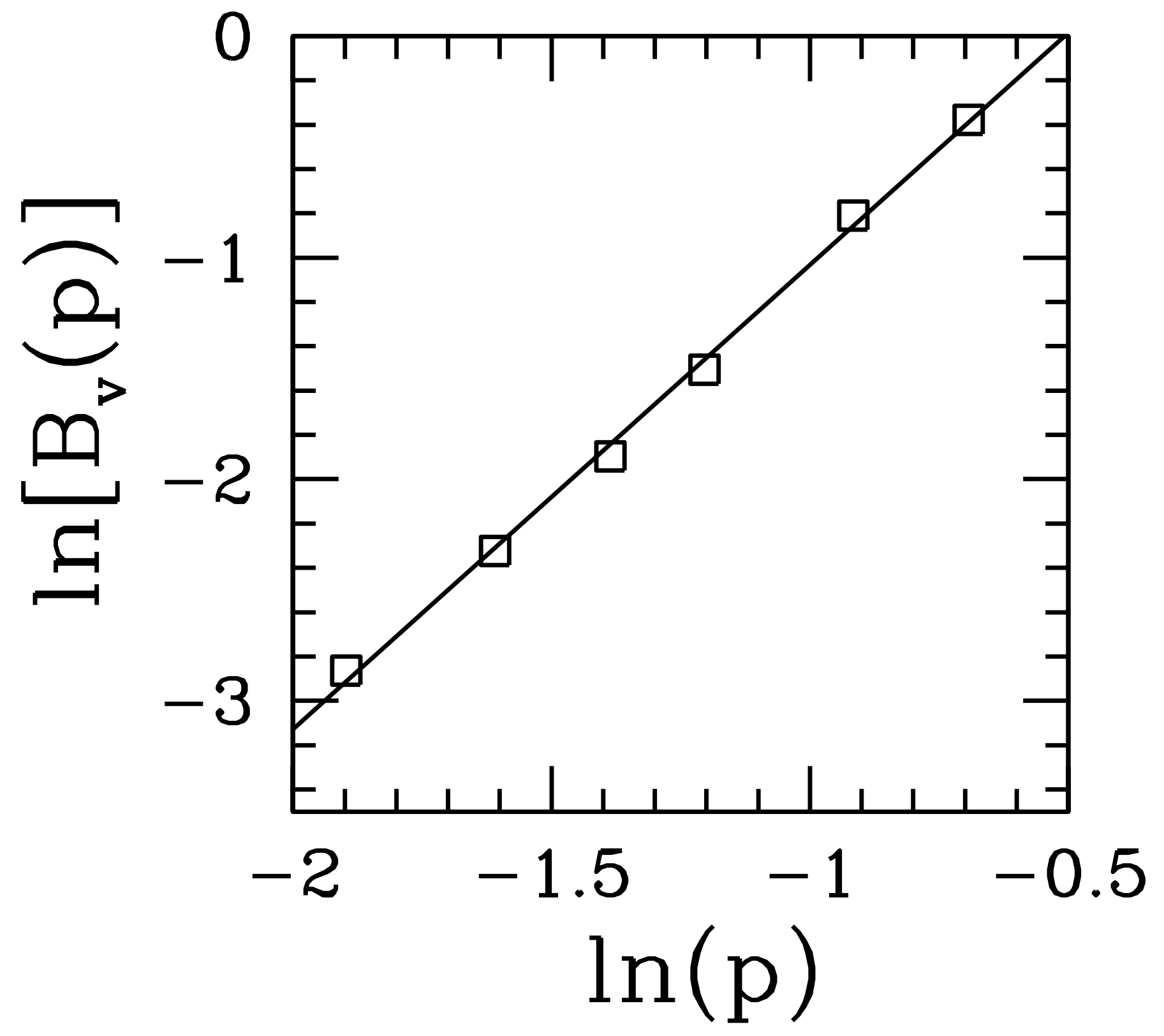



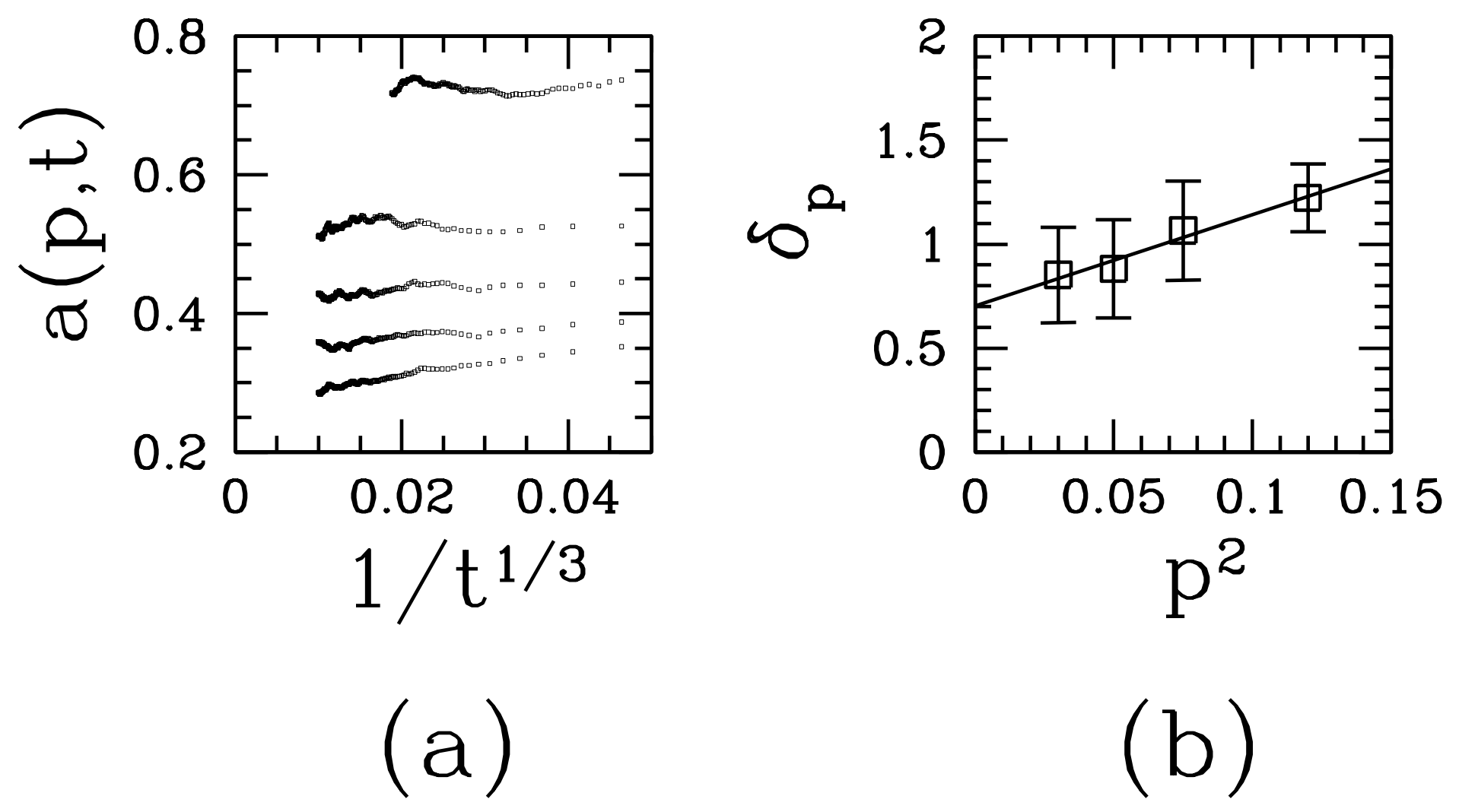

(b) 


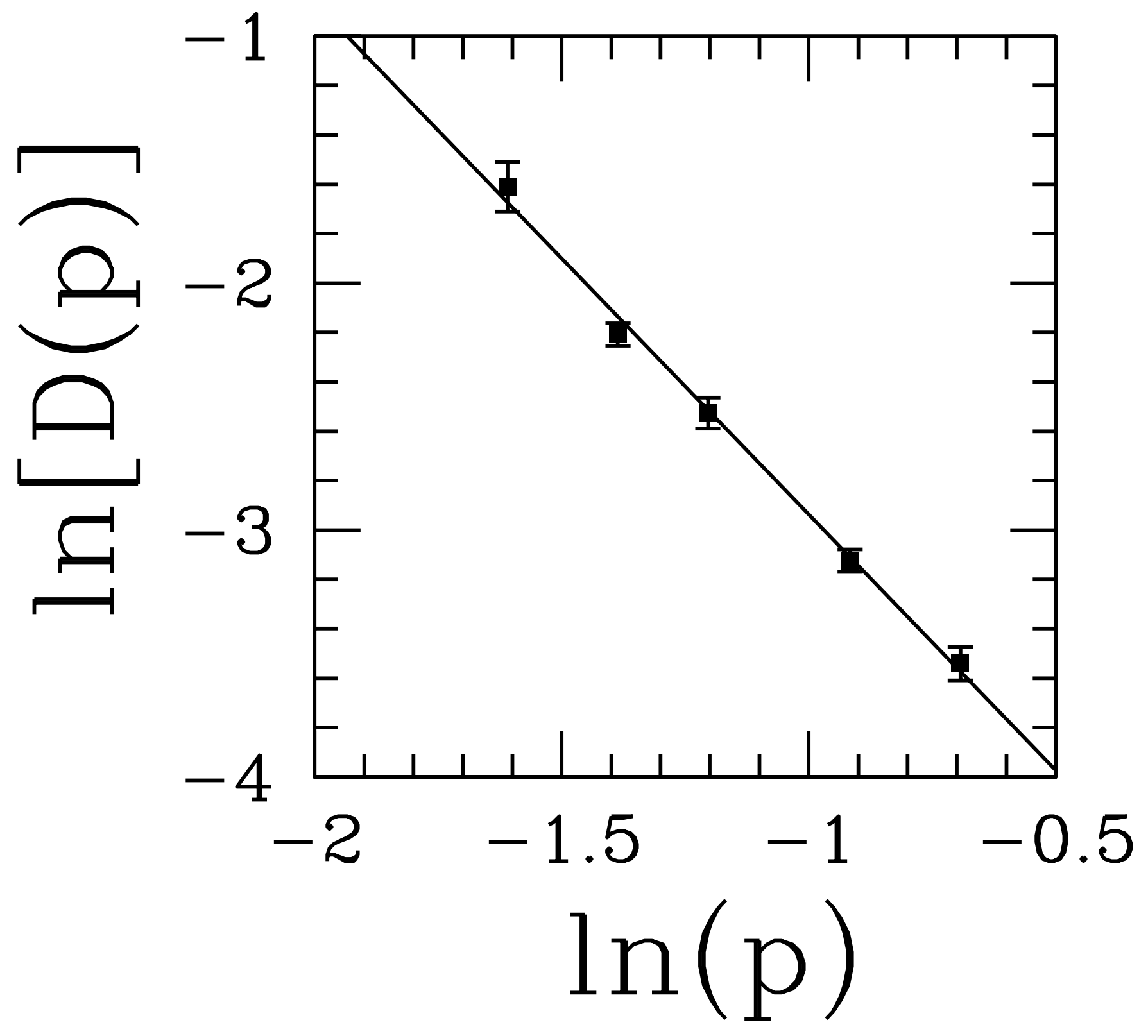




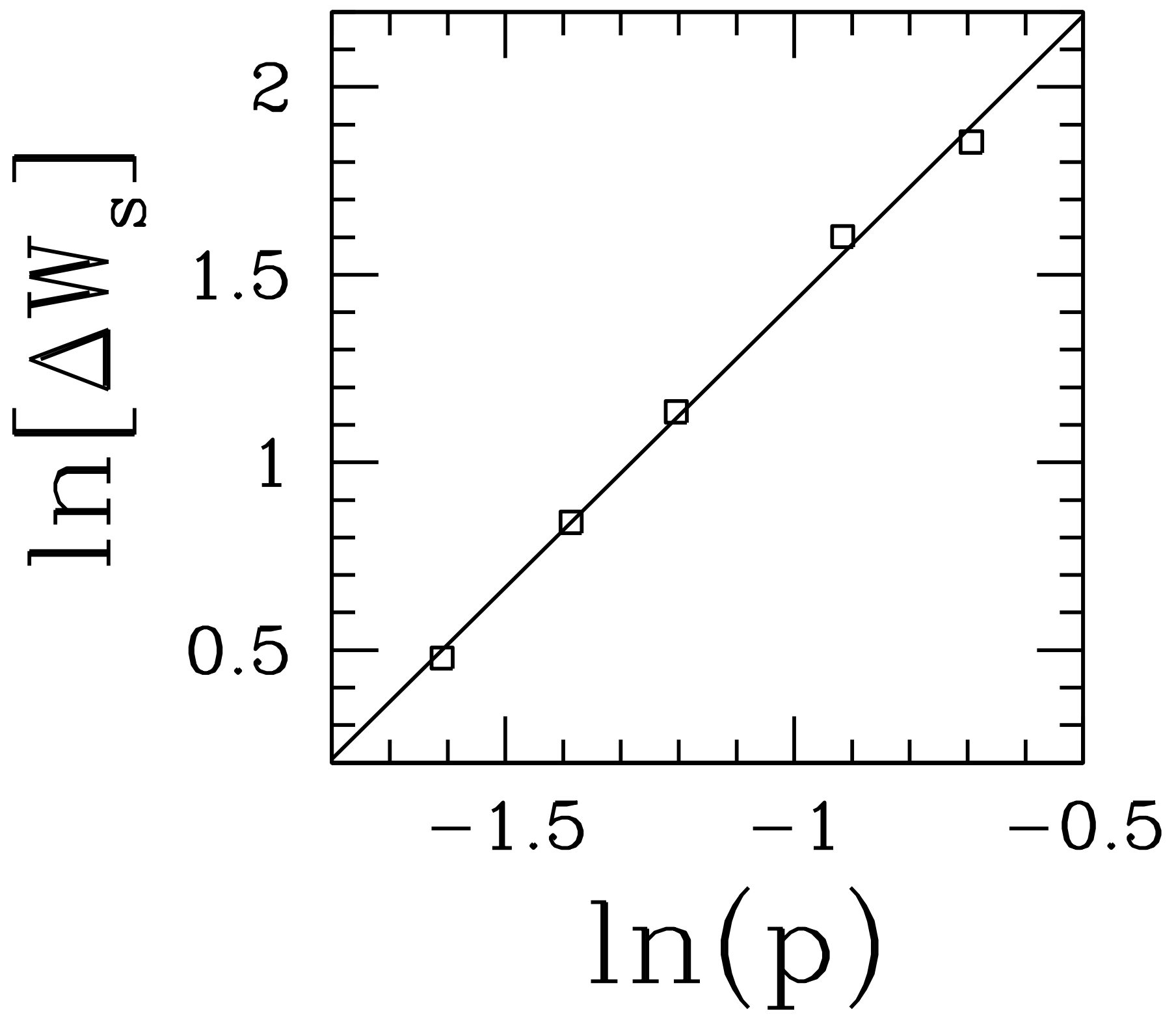

